\title{
Bronchoscopic diagnosis of sarcoidosis
}

\author{
C. Leonard*, V.J. Tormey*, C. O'Keane**, C.M. Burke*
}

Bronchoscopic diagnosis of sarcoidosis. C. Leonard, V.J. Tormey, C. O'Keane, C.M. Burke. CERS Journals Ltd 1997.

ABSTRACT: Transbronchial lung biopsy (TBLB), transbronchial needle aspiration (TBNA) of mediastinal lymph nodes and bronchoalveolar lavage (BAL) are routinely performed at fibreoptic bronchoscopy. Up to the present time, no data have been available on the efficacy of performing all three of these procedures simultaneously in the bronchoscopic work-up of sarcoidosis. A prospective study was undertaken to compare the diagnostic yield from TBLB, TBNA and BAL in patients presenting with clinical and radiological features typical of sarcoidosis.

Thirteen consecutive patients with clinical and radiological features consistent with stage I and II sarcoidosis underwent bronchoscopy with TBLB, TBNA and BAL.

Noncaseating granulomata (stain and culture negative for tuberculosis bacilli and fungi) were found in seven of the 13 patients by TBLB, and in six of the 13 patients by TBNA (of which four patients had negative TBLB). Eight of the 13 patients had classical "sarcoid" BAL findings, i.e. >12\% lymphocytes, and high CD4+:CD8+ lymphocyte ratio. Combining TBLB, TBNA and BAL gave a diagnostic sensitivity of $100 \%$ (12 out of 12 patients) for sarcoidosis. The remaining patient had nondiagnostic bronchoscopic studies and mediastinoscopy biopsy showed a non-Hodgkin's lymphoma.

Our data suggest that performing simultaneous transbronchial lung biopsy, transbronchial needle aspiration and bronchoalveolar lavage produces optimal results in the diagnosis of sarcoidosis.

Eur Respir J 1997; 10: 2722-2724.
*Dept of Respiratory Medicine, James Connolly Memorial Hospital Blanchardstown, Dublin, Ireland. **Dept of Histopathology, Mater Misericordiae Hospital, Dublin, Ireland.

Correspondence: C.M. Burke

Dept of Respiratory Medicine

James Connolly Memorial Hospital

Blanchardstown

Dublin 15

Ireland

Keywords: Sarcoidosis

transbronchial lung biopsy

transbronchial needle aspiration

Received: December 301996

Accepted after revision September 81997

C.L. and V.J.T are supported by British Council Chevening Research Fellowships.
Sarcoidosis is a multisystem granulomatous disease of unknown aetiology, with an incidence of 15-40 per 100,000 of the population per year [1]. The commonest mode of presentation is hilar and mediastinal lymphadenopathy on a chest radiograph [2]. A tissue diagnosis is desirable both to exclude other conditions, which may mimic sarcoidosis [1, 3-7], and as a basis for management decisions, in particular the use of oral corticosteroids.

Transbronchial lung biopsy (TBLB), via a fibreoptic bronchoscope (FOB), is the usual method of obtaining a tissue diagnosis $[1,7,8]$. The presence of noncaseating granuloma in the biopsy specimen is considered diagnostic of sarcoidosis if the tissue is stain- and culture-negative for tuberculosis bacilli and fungi [1]. However, TBLB carries significant complications; in particular, there is a risk of pneumothorax $(1-10 \%)$, and moderate $(>25 \mathrm{~mL})$ to severe $(>100 \mathrm{~mL})$ haemorrhage occurs in $5.4-0.6 \%$ of cases [8], although it is rarely life-threatening $[1,8]$. Furthermore, the diagnostic yield with TBLB in sarcoidosis ranges $40-90 \%$, depending on the skill of the bronchoscopist, the number of biopsies taken, and degree of interstitial involvement at the time of biopsy $[1,9,10]$. Because of this variable yield from TBLB, alternative diagnostic modalities, such as mediastinoscopy or open lung biopsy, may be required to obtain a tissue diagnosis, with the associated risks and costs of a procedure requiring hospital admission and general anaesthesia.

Bronchoalveolar lavage (BAL) is helpful in the diagnosis of sarcoidosis [11-14], but even the European Society of Pneumology Task Group on BAL note that no single feature in BAL is diagnostic of sarcoidosis, and state rather conservatively that the combination of a lymphocytosis with a CD4+:CD8+ ratio $>3.5$ would be consistent with sarcoidosis in an appropriate clinical setting [15]. Other work has shown that whilst a CD4+:CD8+ ratio of S4:1 on BAL has a positive predictive value of $94 \%$ in distinguishing sarcoid from other interstitial lung disease, the sensitivity is only $59 \%$ [12], confirming that BAL alone is not sensitive enough for use as the sole diagnostic tool in the diagnosis of sarcoidosis.

Transbronchial needle aspiration (TBNA), a technique first used in 1949 through a rigid bronchoscope [16], was modified more recently for use through the FOB and is used to sample hilar and mediastinal lymph nodes [17]. TBNA is a safe and effective method of obtaining cores of tissue for the histological diagnosis of benign and malignant disease in the mediastinal and hilar lymph nodes, and for staging malignant disease [9, 10, 17-24]. Some authorities suggest that TBNA may become the procedure of choice for the diagnosis of stage I and II sarcoidosis $[17,19]$. In a series of 51 patients with suspected sarcoidosis, the diagnostic yield was $60 \%$ from TBLB and $53 \%$ from TBNA in stage I disease [10]. Combining TBLB and TBNA increased the diagnostic yield to $83 \%$. In stage II disease, $76 \%$ had the diagnosis confirmed by TBLB and $48 \%$ by TBNA. Combining TBLB and TBNA increased the yield to $86 \%$. 
Up to the present time, there have been no data available on the simultaneous use of the three procedures of TBLB, BAL and TBNA. We therefore wished to investigate 13 consecutive patients with radiological features of stage I or II sarcoidosis in this way.

\section{Materials and methods}

\section{Subjects}

Thirteen consecutive subjects with clinical history, physical findings and chest radiograph consistent with stage I or II sarcoidosis were entered into the study. All patients had a chest radiograph, high-resolution computed tomography (HRCT) scan, full blood count (FBC), coagulation screen and serum calcium prior to entry into the study.

\section{Bronchoscopy}

Patients attended the day ward and were premedicated with $10 \mathrm{mg}$ diazepam, and lignocaine local anaesthesia was used. An Olympus BF10 FOB (Tokyo, Japan) was then inserted nasally, with continuous monitoring of oxygen saturation and cardiograph tracing during the procedure.

\section{Bronchoalveolar lavage}

The bronchoscope was wedged in the right middle lobe bronchus, and three aliquots $(180 \mathrm{~mL})$ of sterile $0.9 \%$ saline instilled through the biopsy channel. After $10 \mathrm{~s}$, the fluid was recovered under suction into a plastic container. The percentage of the lavage fluid recovered ranged 50$65 \%$. The BAL fluid was analysed for total cell count, differential count and CD4+:CD8+ ratio (using fluorescence-labelled monoclonal antibodies and a flow cytometer).

\section{Transbronchial needle aspiration}

The needle used for TBNA was an MW-319 (Mill-Rose Laboratories Inc., Mentor, OH, USA), a $140 \mathrm{~cm}$ long flexible plastic catheter with a retractable 19-gauge metal needle at the distal end. Inside the 19-gauge needle was a 21-gauge needle which was also retractable.

The catheter was advanced through the FOB, with both needles retracted. When the metal hub appeared through the distal end of the FOB, both needles were fully advanced and the tracheal or bronchial wall was pierced. Lymph node tissue was sampled in a standard fashion [17] by advancing, partially withdrawing, and readvancing the needle several times under suction. The areas sampled were the right paratracheal, aortopulmonary window, and anterior and posterior carinal lymph node stations. CT scans were not used to map the areas to be sampled. Once obtained, the TBNA specimen was flushed into formalin for histological examination.

\section{Transbronchial lung biopsy}

Alligator forceps were used via the FOB to obtain four or five biopsies from the right lower lobe in a standard fashion [8].

\section{Results}

No complications were seen in the patients studied, and all were discharged home from the day ward within $4 \mathrm{~h}$
Table 1. - Results of TBLB, TBNA, BAL and HRCT scan in the patients studied

\begin{tabular}{|c|c|c|c|c|c|}
\hline \multirow{2}{*}{$\begin{array}{l}\text { Patient } \\
\text { No. }\end{array}$} & \multirow{2}{*}{ TBLB } & \multirow{2}{*}{ TBNA } & \multicolumn{2}{|c|}{ BAL } & \multirow{2}{*}{$\begin{array}{l}\text { HRCT } \\
\text { scan }^{\dagger \dagger}\end{array}$} \\
\hline & & & $\begin{array}{l}\% \\
\text { lym }\end{array}$ & $\begin{array}{c}\text { CD4+l } \\
\text { CD8+ }\end{array}$ & \\
\hline 1 & -ve & $+\mathrm{ve}$ & 4 & $1.5 / 1$ & Normal parenchyma \\
\hline 2 & $+\mathrm{ve}$ & $-\mathrm{ve}$ & 85 & $5.5 / 1$ & Normal parenchyma \\
\hline 3 & $-\mathrm{ve}$ & -ve & 84 & $8 / 1$ & Normal parenchyma \\
\hline 4 & $+\mathrm{ve}$ & $-\mathrm{ve}$ & 2 & $2 / 1$ & Early interstitial disease \\
\hline $5^{\ddagger}$ & -ve & $-\mathrm{ve}$ & 0.5 & $18 / 1$ & Normal parenchyma \\
\hline 6 & -ve & $+\mathrm{ve}$ & $<1$ & $1.3 / 1$ & Interstitial disease \\
\hline 7 & +ve & -ve & 3 & $2.5 / 1$ & Interstitial disease \\
\hline 8 & -ve & +ve & 18 & $6 / 1$ & Interstitial disease \\
\hline 9 & $+\mathrm{ve}$ & $-\mathrm{ve}$ & 36 & $7.5 / 1$ & Normal parenchyma \\
\hline 10 & $+\mathrm{ve}$ & $+\mathrm{ve}$ & 25 & $20 / 1$ & Interstitial disease \\
\hline 11 & $-\mathrm{ve}$ & $+\mathrm{ve}$ & 76 & $17 / 1$ & Interstitial disease \\
\hline 12 & $+\mathrm{ve}$ & $+\mathrm{ve}$ & 36 & $9 / 1$ & Interstitial disease \\
\hline 13 & $+\mathrm{ve}$ & $-\mathrm{ve}$ & 41 & $6 / 1$ & Interstitial disease \\
\hline
\end{tabular}

A positive TBNA or TBLB required the finding of noncaseating granulomata on histological examination, with all tissue negative for tuberculosis and fungal stains. $*$ : patient No. 5 had negative TBLB, TBNA and BAL (mediastinoscopic lymph node biopsy confirmed non-Hodgkin's lymphoma); tt: all patients had hilar and mediastinal lymphadenopathy as defined by entry criteria to study. -ve: negative, i.e. nondiagnostic; +ve: positive, i.e. diagnostic; TBLB: transbronchial lung biopsy; TBNA: transbronchial needle aspiration; BAL: bronchoalveolar lavage; lym: lymphocytes; HRCT: high-resolution computed tomography.

of the bronchoscopy. Postbronchoscopy chest radiographs showed no evidence of pneumothorax in any of the cases.

The results are presented in table 1. The TBLB was positive in seven patients (i.e. showed noncaseating granulomata, which were stain- and culture-negative for tuberculosis bacilli and fungi), five of whom also had a positive BAL [7] (lymphocytosis $>12 \%$ with a CD4+:CD8+ ratio $>4: 1$ ). TBNA was positive in six patients, of whom two also had a positive TBLB and BAL and two had a positive BAL alone. Therefore, two patients were diagnosed solely by TBNA and two solely by TBLB.

Two patients had negative TBLB and TBNA. One of these had a typical "sarcoid" BAL (Subject No. 3 in table 1), with $84 \%$ lymphocytes and a CD4+:CD8+ ratio of 8:1, and a diagnosis of sarcoidosis was made on this basis. This patient did not receive any corticosteroid medication and the chest radiograph had returned to normal 3 months later.

The final patient (Subject No. 5) had negative TBNA and TBLB and normal BAL findings. A mediastinoscopic lymph node biopsy showed non-Hodgkin's lymphoma.

Therefore, simultaneous use of the three diagnostic modalities of TBLB, TBNA and BAL successfully diagnosed sarcoidosis in all of the 12 with this diagnosis and prevented the need for more invasive procedures.

Chest radiography and HRCT scan demonstrated lymphadenopathy in all cases, and showed evidence of interstitial disease in eight patients. Of these eight, only five had a positive TLB. Five patients had no evidence of interstitial disease on chest radiography or HRCT, but two of these had positive TBLB.

\section{Discussion}

Thirteen consecutive patients with clinical and radiological features consistent with stage I or II sarcoidosis 
were studied. The data show that combining the modalities of TBLB, TBNA and BAL achieved significantly better results than either TBLB, TBNA or BAL alone. Furthermore, the significantly enhanced diagnostic yield from combining these three procedures at the same bronchoscopy sitting was not associated with any additional complications. TBLB was diagnostic in seven out of 12 patients with sarcoidosis (58\%), five of whom had interstitial lung disease on HRCT scan. However, TBLB was positive in two patients in whom HRCT scan showed normal lung parenchyma. These data are consistent with previous reports, which show that HRCT scanning is not useful in predicting the diagnostic yield of TBLB $[25,26]$. Although our yield of $58 \%$ from TBLB is comparable to previous reports, five of the 12 patients with sarcoidosis had negative TBLB, and in the absence of TBNA and BAL at least some of these patients might have required the significantly more risky and costly procedures of mediastinoscopy or open lung biopsy to confirm the diagnosis.

TBNA can successfully sample lymph glands in a variety of pathological states. There are few reports of complications following this procedure and only a single case report records a significant complication, namely bacterial pericarditis following TBNA [27]. In four of the present patients with a negative TBLB, TBNA confirmed the diag-nosis of sarcoidosis and obviated the necessity for consideration of more invasive procedures in these patients, with obvious cost-benefit implications. The overall diagnostic yield from TBNA in patients with sarcoidosis was $50 \%$ (six out of 12 patients) and is consistent with previous reports. None of the patients had any complications from the TBNA procedure.

With regard to BAL, the present data are consistent with previous reports on the utility of BAL in the diagnosis of sarcoidosis; four of the 11 patients with positive biopsies had negative BAL, underlining the poor sensitivity of BAL in sarcoidosis. However, combining BAL, TBLB and TBNA gave a $100 \%$ diagnostic yield for those patients with sarcoidosis.

We are not aware of any existing information which addresses the usefulness of combining bronchoalveolar lavage, transbronchial needle aspiration and transbronchial lung biopsy at the same bronchoscopy sitting in the diagnosis of sarcoidosis. Our results suggest that the three procedures can be safely and effectively carried out at a single bronchoscopy sitting with enhanced diagnostic yield, little additional cost and no additional complications in patients with sarcoidosis.

\section{References}

1. Seaton A, Seaton D, Leitch AG. In: Crofton and Douglas's Respiratory Diseases. 4th Edn. Oxford, Blackwell Scientific Publications, 1989; pp. 630-659.

2. De Remee RA. Sarcoidosis. Mayo Clinic Proc 1995; 70: 177-181.

3. Fazzi P, Solfanelli S, Morelli G, et al. Sarcoidosis: single bulky mesenteric lymph node mimicking a lymphoma. Sarcoidosis 1995; 12: 75-77.

4. Badr AI, Sharma OP. Ki-1 anaplastic large cell lymphoma: another masquerader of sarcoidosis. A case report. Sarcoidosis 1993; 10: 62-65.

5. Blackmon GM, Raghu G. Pulmonary sarcoidosis: a mimic of respiratory infection. Semin Respir Infect 1995; 10: 176-186.

6. Hoheisel GB, Chan BK, Chan CH, Costabel U. Mimics of sarcoidosis: endobronchial tuberculosis. Sarcoidosis 1993; 10: 9-11.

7. Fanburg BL, Lazarus DS. Sarcoidosis. In: Murray JF, Nadel JA, eds. Textbook of Respiratory Medicine. 2nd Edn. Philadelphia, W.B. Saunders Co., 1994; pp. 18731888.

8. McDougall JC, Cortese DA. Bronchoscopic lung biopsy. In: Prakash UBS, ed. Bronchoscopy. New York, Raven Press, 1994; pp. 141-146.

9. Pauli G, Pelletier A, Bohner C, Roeslin N, Warter A, Roegel E. Transbronchial needle aspiration in the diagnosis of sarcoidosis. Chest 1984; 85: 482-484.

10. Morales CF, Patefield AJ, Strollo PJ Jr, Schenk DA. Flexible transbronchial needle aspiration in the diagnosis of sarcoidosis. Chest 1994; 106: 709-711.

11. Drent M, Mulder PG, Wagenaar SS, Hoogsteden HC, van Velzen-Blad H, van den Bosch JM. Differences in BAL fluid variables in interstitial lung diseases evaluated by discriminant analysis. Eur Respir J 1993; 6: 803-810.

12. Winterbauer RH, Lammert J, Selland M, et al. Bronchoalveolar ravage cell populations in the diagnosis of sarcoidosis. Chest 1993; 104: 352-361.

13. James DG, Rizzato G, Sharma OP. Bronchopulmonary lavage (BAL): a window of the lungs. Sarcoidosis 1992; 9: 3-14.

14. Milman N, Graudal N, Jacobsen GK. Bronchoalveolar lavage in radiologically detected diffuse lung disease: diagnostic value of total and differential cell count in a series of 130 patients. APMIS 1995; 103: 764-768.

15. Poulter LW, Rossi GA, Bjermer L, et al. The value of bronchoalveolar ravage in the diagnosis and prognosis of sarcoidosis. Eur Respir J 1990; 3: 943-944.

16. Schieppati E. Mediastinal lymph node puncture through the tracheal carina. Surg Gynecol Obstet 1958; 110: 243 246.

17. Wang KP. Flexible transbronchial needle aspiration biopsy for histologic specimens. Chest 1985; 88: 860863.

18. Haponik EF, Capellari JO, Chin R, et al. Education and experience improve transbronchial needle aspiration performance. Am J Respir Crit Care Med 1995; 151: 1998 2002.

19. Mehta A, Kavuru MS, Meeker DP, et al. Transbronchial needle aspiration for histology specimens. Chest 1989; 96: 1228-1232.

20. Salathe M, Soler M, Bolliger CT, et al. Transbronchial needle aspiration in routine fibreoptic bronchoscopy. Respiration 1992; 59: 5-8.

21. Schenk DA, Chambers SL, Derdak S, et al. Comparison of the Wang 19-gauge and 22-gauge needles in the mediastinal staging of lung cancer. Am Rev Respir Dis 1993; 147: 1251-1258.

22. Baron KM, Aranda CP. Diagnosis of mediastinal mycobacterial lymphadenopathy by transbronchial needle aspiration. Chest 1991; 100: 1723-1724.

23. Harrow E, Halber M, Hardy S, Halteman W. Bronchoscopic and roentgenographic correlates of a positive transbronchial needle aspiration in the staging of lung cancer. Chest 1991; 100: 1592-1596.

24. Midthun DE, Cortese DA. Bronchoscopic needle aspiration and biopsy. In: Prakash UBS, ed. Bronchoscopy. New York, Raven Press, 1994; pp. 147-154.

25. Miller BH, Rosado-de-Christenson ML, McAdams HP, Fishback NF. Thoracic sarcoidosis: radiologic-pathologic correlation. Radiographics 1995; 15: 421-437.

26. Mana J, Teirstein AS, Mendelson DS, et al. Excessive thoracic computed tomographic scanning in sarcoidosis. Thorax 1995; 50: 1264-1266.

27. Epstein SK, Winslow CJ, Brecher SM, et al. Polymicrobial bacterial pericarditis after transbronchial needle aspiration: case report with an investigation on the risk of bacterial contamination during fiberoptic bronchoscopy. Am Rev Respir Dis 1992, 146: 523-525. 